Politikmuster und Innovationswirkungen der Umweltpolitik im internationalen Vergleich

\section{Muster mit Wert}

\section{Eindimensionale Erklärungsmuster können den komplexen und interdependenten Einflüssen der Umweltpolitik auf Innovationen nicht gerecht werden. Notwen- dig isł vielmehr ein Analyseraster, das umweltökonomische, innovationsökono- mische und politikanalytische Forschungsansätze vereint. Hierzu wird das Kon- zept des Politikmusters entwickelt und auf Fallstudien in verschiedenen Sekforen und Ländern angewendet.}

$\mathrm{F}$ Von Jïrgen Blazejczak, Dietmar Edler, Jens Hemmelskamp und Martin Jänicke ür eine langfristige Sicherung der natürlichen Ressourcen und der Umweltqualität im Kontext einer nạchhaltigen Entwicklung erscheinen grundlegende Veränderungen der bestehenden Produktionsweisen und Lebensstile notwendig. Umweltinnovationen verringern die Kosten von Strategien zur Reduzierung der Inanspruchnahme natürlicher Ressourcen und erleichtern so die Erreichung von Nachhaltigkeitszielen. Für die Politik stellt sich somit die Frage, wie eine Richtungsänderung der Innovationsaktivitäten hin zu mehr Umweltinnovationen erreicht werden kann.

Eine gemeinsame Studie des Deutschen Instituts für Wirtschaftsforschung (DIW), der Forschungsstelle für Umweltpolitik der Freien Universität Berlin (FFU) und des Zentrums für Europäische Wirtschaftsforschung (ZEW) untersucht anhand internationaler Fallstudien die Frage, wie die Umweltpolitik im Hinblick auf eine Förderung von Umweltinnovationen gestaltet werden sollte (1). Betrachtet werden dabei die Sektoren Papier- und Zellstoffherstellung (Japan, Schweden, USA), Windenergieerzeugung (Dänemark, Deutschland, Großbritannien) und Herstellung elektrischer Haushaltsgeräte (Dänemark). Der Innovationserfolg wird entweder als Diffusionsrate einer neuen Technologie oder als Umweltentlastung durch eine Reihe von eher inkrementellen technischen Neuerungen gemessen. In dieser Studie wird nicht versucht, die Innovationswirkungen einzelner umweltpolitischer Instrumente zu isolieren. Vielmehr wird davon ausgegangen, daß aufgrund der hohen Komplexität und Dynamik von Einflußfaktoren, die Innovationsprozesse auszeichnen, diese nur durch das Zusammenwirken mehrerer Einflußfaktoren erklärt werden können.
Die neoklassische Umweltökonomie unternimmt den Versuch, die Innovationswirkungen einzelner umweltpolitischer Instrumente isoliert zu analysieren, in erster Linie unter dem Kriterium der dynamischen Effizienz. Als Ergebnis wird überwiegend der Einsatz sogenannter Marktlösungen wie Abgaben oder Zertifikate zur umweltpolitischen Regulierung empfohlen. Neuere Erkenntnisse verschiedener Gebiete der ökonomischen Theorie, z.B. der Innovationsökonomie, der Neuen Institutionellen Ökonomik und der Evolutionsökonomie, wie auch die Ergebnisse der vergleichenden Politikforschung legen jedoch einen breiteren Forschungs- und Analyseansatz nahe, weil eindimensionale Erklärungsmuster den komplexen und interdependenten Einflüssen der Umweltpolitik auf Innovationen nicht gerecht werden können. Deshalb wird in der Studie ein Analyseraster entwickelt, das in einer Synthese umweltökonomische, innovationsökonomische und politikanalytische Forschungsansätze und Kategorien vereint.

\section{Kennzeichen innovations- freundlicher Politikmuster}

Zentraler analytischer Begriff ist das Konzept des Politikmusters. Als Politikmuster wird die Gesamtheit aller kalkulierbaren Regeln, Vorgehensweisen und Handlungskontexte in einem Gegenstandsbereich staatlicher Einflußnahme verstanden, wobei auch das Spektrum von Instrumenten, die positive Anreize setzen, ausdrücklich mit eingeschlossen wird.

Es lassen sich drei zentrale Dimensionen identifizieren, die in ihrer Ausgestaltung die Innovationsfreundlichkeit eines Politikmusters beeinflussen:

- die Instrumentierung, die über die Auswahl der einzelnen Instrumente hinaus auch deren Gestaltung und Kombination umfaßt, der Politikstil, der die Art der Zielbildung und der Implementierung umweltpolitischer Regulierungen beschreibt, und

- die Akteurskonfiguration, die die institutionellen Rahmenbedingungen und das Netzwerk der Akteure bezeichnet.

Durch die Ausdifferenzierung dieser unterschiedlichen Dimensionen entsteht ein Analyseraster, das zur Beurteilung der Politikmuster der untersuchten Fallstudien in den jeweils betrachteten Ländern dient.

Als Ergebnis der Auswertung der Erkenntnisse von Umweltökonomie, Innovationsökonomie und Politikanalyse lassen sich die Elemente eines innovationsfreundlichen Politikmusters folgendermaßen charakterisieren:

Eine Instrumentierung ist innovationsfreundlich, wenn sie...

ökonomische Anreize setzt,

- mehrere Instrumente kombiniert,

auf strategischer Planung und Zielbildung basiert und

- Innovation als Prozeß sowie und die verschiedenen Innovationsphasen berïcksichtigt.

Ein Politikstil ist innovationsfreundlich, wenn er...

dialogisch und konsensual,

- kalkulierbar, verlässlich und kontinuierlich,

- entschlossen, proaktiv und anspruchsvoll,

- lernoffen und flexibel in bezug auf den Einzelfall und

- managementorientiert und wissensbasiert ist. Eine Akteurskonfiguration ist innovationsfreundlich, wenn...

- sie Politikintegration horizontal und vertikal begünstigt, und wenn die Vernetzung zwischen verschiedenen Regulierungsinstanzen eng ist,

- verschiedene Regulierungsadressaten miteinander vernetzt sind,

- die Vernetzung zwischen Regulierern und Regulierten eng ist und wenn

- sie durch die Einbeziehung von ,stakeholdern" in Akteursnetzwerke dezentral vorhandenes Wissen und Motivation verfügbar macht.

\section{Anwendung auf die Fallstudien}

Bei der Auswertung dieses Rasters zur Interpretation der untersuchten sieben Politikmuster sind wegen des explorativen Charakters der Studie, aber auch aufgrund der geringen Zahl und des unterschiedlichen Zuschnitts der Fallstudien, Einschränkungen zu machen. Weitgehende Schlußfolgerungen lassen sich zum einen nicht ziehen, weil nicht in allen Fällen 
spezifische Innovationswirkungen (als explanandum) einem ebenso spezifischen Politikmuster (als explanans) zuzuordnen waren. Dies gilt für die drei Fälle der Papierindustrie, bei denen das Politikmuster auf mehrere, auch inkrementale Verbesserungen bezogen ist. Zum anderen ist ein klares Ranking des Innovationsgrades der sieben Fälle nicht möglich. Allenfalls lassen sich die beiden dänischen Fälle (Elektrogeräte, Windenergie) als Beispiele relativ weitgehender - zudem spezifischer Innovationen verstehen, und zwar auch im Sinne internationaler Pionierleistungen. Auch die im internationalen Vergleich weitgehenden Innovationsaktivitäten der schwedischen Papierindustrie lassen sich in diesem Sinne interpretieren, ergeben also einen „Fall“ im Sinne der Profilierung innerhalb einer Grundgesamtheit (hier der Vergleichsländer).

\section{- Erfolg "runder" Politikmuster}

Die Analyse der Fallstudien legt jedoch die Schlußfolgerung nahe, daß den weitestgehenden Innovationserfolgen auch das jeweils ,rundeste" Politikmuster im Sinne eines breiten Sets innovationsfördernder Einflußfaktoren entspricht. Insoweit stiitzen die untersuchten Fälle die Hypothese, daß politisches Handeln der Dynamik und Komplexität der Einflußfaktoren von Innovationsprozessen am ehesten mit einem breiten, instrumentell nicht restringierten Ansatz gerecht wird. Gemeinsam sind den Politikmustern in diesen Erfolgsfällen insbesondere der strategische Ansatz, kombiniert mit einer hohen Zielstrebigkeit, und die starke Bedeutung von Netzwerken.
Im Fall der deutschen Windenergieerzeugung zeigt sich, daß auch ein „engeres“ Politikmuster mit einer starken Wirkung ökonomischer Anreizmechanismen erfolgreich sein kann. Da aber Strategien der Innovationsförderung ex ante durch Unsicherheit über die fallspezifischen Wirkungsfaktoren gekennzeichnet sind, empfiehlt sich generell ein breiterer Ansatz mit einem Mix von Instrumenten, dialogischem Vorgehen, starker Vernetzung der beteiligten Akteure usw. Für einen breiteren Ansatzes spricht auch, daß die Innovationswirkungen auf diese Weise auch ohne das Überstrapazieren eines einzelnen Instruments erzielt werden können.

Insgesamt bestätigen die behandelten sieben Fallstudien die Hypothese, daß Innovationswirkungen politischer Maßnahmen mit einem einfachen, linearen Top-down-Schema weder erklärbar noch realisierbar sind. Dagegen läßt sich - unter den skizzierten Einschränkungen folgern, daß eine innovationsorientierte Umweltpolitik umfassende Anstrengungen verlangt, die eher in Kategorien des strategischen ,public management" als in Kategorien eines auf einzelne Instrumente fixierten und auf formale Regeln aufbauenden traditionellen Verwaltungshandelns $\mathrm{zu}$ konzipieren sind. Neuere Ansätze der Umweltpolitik in einigen Ländern (Schweden, USA) tragen diesem Erkenntnisstand bereits Rechnung.

\section{Anmerkung}

(1) Vgl. für eine ausführlichere Darstellung Blazejczak, J./ D. Edler/ J. Hemmelskamp/ M. Jänicke: Umweltpolitik und Innovation: Politikmuster und Innovationswirkungen im internationalen Vergleich, Zeitschrift für Umweltpolitik \& Umweltrecht, 1/99, S. 1-32.

Zu den einzelnen Teilstudien, die im Rahmen des Forschungsverbundes "Innovative Wirkungen umweltpolitischer Instrumente" im Auftrag des Bundesministeriums für Bildung, Forschung und Technologie erstellt wurden, vgl. Blazejczak, J./ D. Edler: Elemente innovationsfreundlicher Politikmuster - ein internationaler Vergleich am Beispiel der Papierindustrie, DIW, Berlin 1998 (vervielfältigtes Manuskript) und

Hemmelskamp, J.: Innovationswirkungen der Umwelt- und Technologiepolitik im Windenergiebereich - ein internationaler Vergleich, ZEW, Mannheim 1998 (vervielfältigtes Manuskript) sowie

Jänicke, M./L. Mez/ P. Bechsgaard/ B. Klemmensen: Innovationswirkungen branchenbezogener Regulierungsmuster am Beispiel energiesparender Kühlschränke in Dänemark, FFU-report 98/3, Berlin, Roskilde 1998.

Zusammenfassungen finden sich in Klemmer, P. (Hrsg.): Innovationen und Umwelt: Follstudien zum Anpassungsverhalten in Wirtschaft und Gesellschaft, Berlin 1999.

\section{Die Autoren}

Dr. Jürgen Blazejczak und Dr. Dieimar Edler sind wissenschaftliche Mitarbeiter am Deutschen Institut für Wirischafisforschung, Berlin, Dr. Jens Hemmelskamp ist wissenschaftlicher Mitarbeiter am Institute for Prospective Technological Studies, Sevilla und Prof. Dr. Martin Jänicke ist Leiter der Forschungsstelle für Umweltpolitik der Freien Universitöt Berlin.

Kontakt: Dietmar Edler, DIW, Posifach, 14191 Berlin. Tel. 030/89789-280, Fax -200,

E-mail: dedler@diw.de

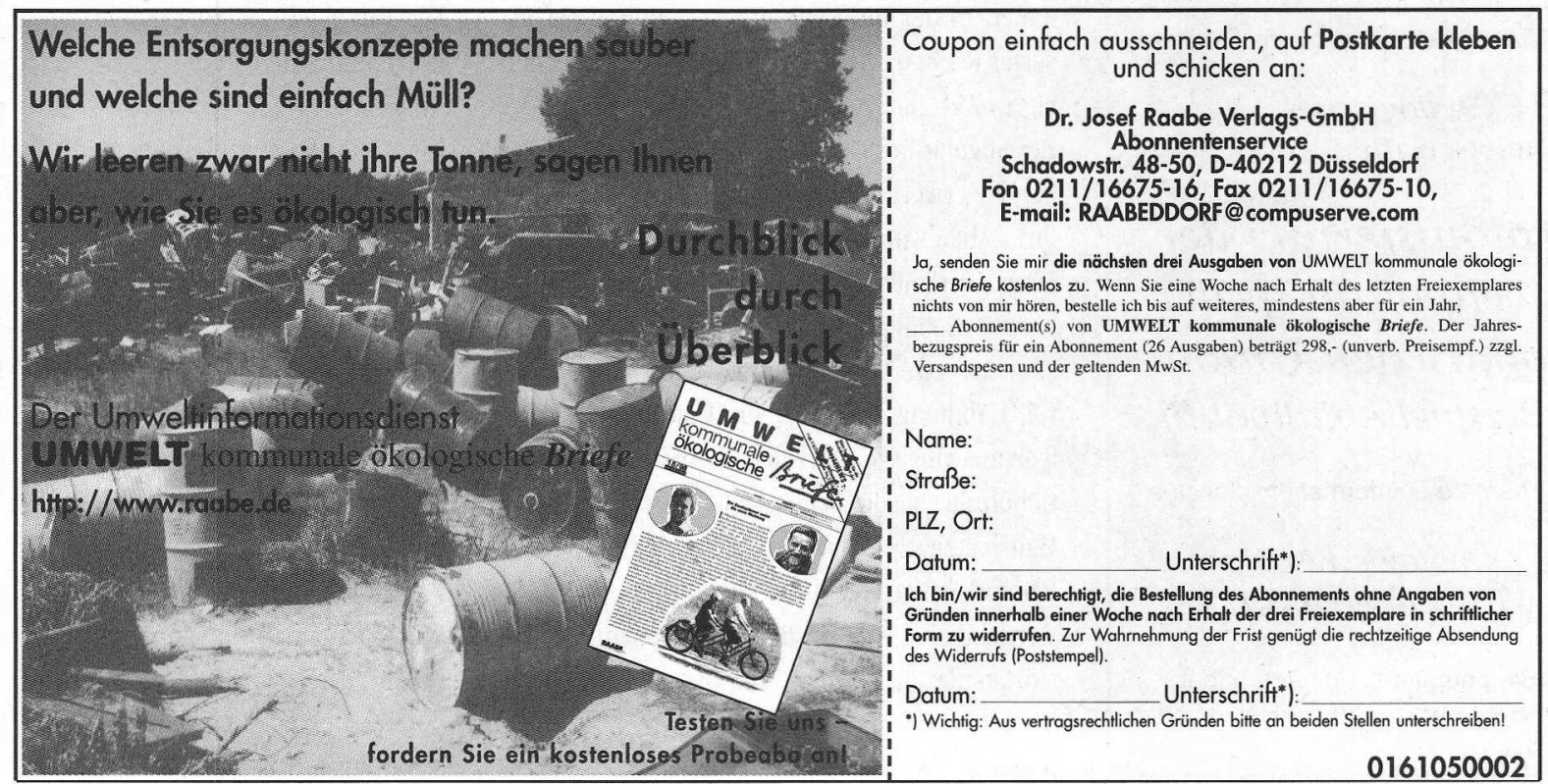


(c) 20I0 Authors; licensee IÖW and oekom verlag. This is an article distributed under the terms of the Creative Commons Attribution Non-Commercial No Derivates License (http://creativecommons.org/licenses/by-nc-nd/3.o/), which permits unrestricted use, distribution, and reproduction in any medium, provided the original work is properly cited. 